\title{
A SURFACE IN ODD CHARACTERISTIC WITH DISCRETE AND NON-FINITELY GENERATED AUTOMORPHISM GROUP
}

\author{
KEIJI OGUISO
}

\begin{abstract}
It was proved by Tien-Cuong Dinh and me that there is a smooth complex projective surface whose automorphism group is discrete and not finitely generated. In this paper, after observing finite generation of the automorphism group of any smooth projective surface birational to any K3 surface over any algebraic closure of the prime field of odd characteristic, we will show that there is a smooth projective surface, birational to some K3 surface, such that the automorphism group is discrete and not finitely generated, over any algebraically closed field of odd characteristic of positive transcendental degree over the prime field.
\end{abstract}

\section{INTRODUCTION}

Let $p$ be an odd prime integer and let $\mathbb{F}_{p}:=\mathbb{Z} /(p)$ be the prime field of characteristic $p$. Let $\mathbb{F}_{p}(t)$ be a purely transcendental extension of degree one of the field $\mathbb{F}_{p}$. We choose and fix an algebraic closure $k_{0}$ of $\mathbb{F}_{p}$ and an algebraically closed field $k$ such that $\mathbb{F}_{p}(t) \subset k$, eg. an algebraic closure of the field $\mathbb{F}_{p}(t)$. Note that any algebraically closed field of characteristic $p$ is isomorphic to either $k_{0}$ or some $k$ defined here. For our purpose, we may and do assume that

$$
\mathbb{F}_{p} \subset \mathbb{F}_{p^{n}} \subset k_{0} \subset k
$$

for all integers $n \geq 1$. Here $\mathbb{F}_{p^{n}}$ is a finite field of cardinality $p^{n}$.

For a variety $V$ defined over a field $K$, we denote the group of the automorphisms of $V$ over $K$ by Aut $(V / K)$ (See also Remark 1.3) and for a field extension $K \subset L$, we denote $V \times_{\text {Spec } K} \operatorname{Spec} L$ by $V_{L}$.

Our main results are Theorem 1.1 and Corollary 1.2 below. Both (1) and (2) in Theorem 1.1 are related to a question posed by [DO19, Problem 1.2]; (2) gives an affirmative answer in any odd characteristic, whereas (1) provides a negative evidence over $k_{0}$.

Theorem 1.1. (1) Let $k_{0}$ be the base field. Then for any smooth projective surface $Y$ birational to a K3 surface over $k_{0}$ and for any field extension $k_{0} \subset L$, the automorphism group $\operatorname{Aut}\left(Y_{L} / L\right)$ is finitely generated.

(2) Let $k$ be the base field. Then there is a smooth projective surface $Y$ birational to some K3 surface such that Aut $(Y / k)$ is not finitely generated.

Theorem 1.1 (1) is a special case of a slightly more general result on finite generation of the discrete automorphism group $\operatorname{Aut}(Y) / \operatorname{Aut}^{0}(Y)$ of a smooth projective surface $Y$ defined over $k_{0}$ (Theorem $2.4(3)$ ). Theorem 2.4 (3) is of its own interest and gives an answer to a question by the referee.

The author is supported by JSPS Grant-in-Aid (S) 15H05738, JSPS Grant-in-Aid (B) 15H03611, KIAS Scholar Program and by NCTS Scholar Program. 
Corollary 1.2. Let $k$ be the base field. Then, for any integer $d$ such that $d \geq 2$, there is a smooth projective variety $Y_{d}$ of $\operatorname{dim} Y_{d}=d$ such that $\operatorname{Aut}\left(Y_{d} / k\right)$ is discrete and not finitely generated.

Remark 1.3. Let $K$ be an algebraically closed field.

(1) Let $V$ be a projective variety defined over $K$. Then the group Aut $(V / K)$ has a natural scheme structure as a locally noetherian subscheme of the Hilbert scheme Hilb $(V \times V)$ under the identification of an automorphism with its graph. We denote by $\operatorname{Aut}^{0}(V / K)$ the connected component containing id $V$. We say that $\operatorname{Aut}(V / K)$ is discrete if $\operatorname{Aut}^{0}(V / K)$ is reduced and $\operatorname{Aut}^{0}(V / K)=\left\{\operatorname{id}_{V}\right\}$. If $V$ is smooth, then $H^{0}\left(V, T_{V}\right)$ is the Zariski tangent space of Aut $(V / K)$ at $\operatorname{id}_{V}$, therefore, Aut $(V / K)$ is discrete if and only if $H^{0}\left(V, T_{V}\right)=0$.

(2) Let $S$ be a K3 surface defined over $K$, that is, a smooth projective surface defined over $K$ with $h^{1}\left(S, \mathcal{O}_{S}\right)=0$ and with a nowhere vanishing global regular 2-form. Then $S_{L}$ is also a K3 surface over $L$ for any field extension $K \subset$ L. Recall that $H^{0}\left(S, T_{S}\right)=0$ also in positive characteristic by [RS76, Theorem 7]. Therefore, $\operatorname{Aut}(S / K)$ is discrete, i.e., $\operatorname{Aut}^{0}(S / K)=\left\{\operatorname{id}_{S}\right\}$. Recall also that Aut $(S / K)=\operatorname{Bir}(S / K)$ by the minimality of the surface $S$. If we have a birational morphism $\tau: T \rightarrow S$ from a smooth projective surface $T$, then we have an inclusion $H^{0}\left(T, T_{T}\right) \subset H^{0}\left(S, T_{S}\right)$ via $\tau$ and therefore Aut $(T / K)$ is also discrete as well. Moreover, Aut $(T / K)$ can be regarded as a subgroup of $\operatorname{Aut}(S / K)=\operatorname{Bir}(S / K)$ via $\tau$ as follows:

$$
\operatorname{Aut}(T / K) \subset \operatorname{Aut}(S / K) ; f \mapsto \tau \circ f \circ \tau^{-1} .
$$

This work is much inspired by recent two remarkable works, due to Lesieutre [Le17] in which a 6-dimensional example as in Theorem 1.1(2), also over characteristic 2, is constructed, and due to Dinh and me [DO19] in which a complex surface example as in Theorem 1.1(2) is finally constructed.

Let $S$ be a K3 surface defined over an algebraically closed field $K$. Sterk St85 shows the finite generation of Aut $(S / K)$ when $K$ is of characteristic zero by using the Torelli theorem for complex K3 surfaces (See also Lemma 2.2). Then Lieblich and Maulik [LM18, Thorem 6.1 and its proof] shows the finite generation of Aut $(S / K)$ when $K$ is of odd characteristic as Theorem 1.4 below. They reduce to characteristic zero when $S$ is not supersingular (Theorem 1.4 (2)), while they use the crystalline Torelli theorem, which is not yet settled in characteristic 2 , when $S$ is supersngular.

Theorem 1.4. Let $S$ be a K3 surface defined over an algebraically closed field $K$ of odd characteristic. Then

(1) $\operatorname{Aut}(S / K)$ is finitely generated.

(2) Assume in addition that $S$ is not supersingular. Then there are a discrete valuation ring $R$ with residue field is $K$ and fraction field $Q(R)$ of characteristic 0 and a smooth projective morphism $\pi: X \rightarrow \operatorname{Spec} R$ with special fiber $S$ such that the specialization map

$$
\operatorname{Aut}(\tilde{S} / \tilde{K}) \rightarrow \operatorname{Aut}(S / K)
$$

has finite kernel and cokernel. Here $\tilde{S}$ is the geometric generic fiber of $\pi$ and $\tilde{K}$ is an algebraic closure of the fractional field $Q(R)$, in particular, $\tilde{S}$ is a K3 surface defined over an algebraically closed field $\tilde{K}$ of charcateristic zero. 
We prove Theorem 1.1 (1) as an application of Theorem 2.4 in Section 2.

Our proof of Theorem 1.1 (2) is quite close to [DO19. As in DO19, we explicitly construct a desired surface $Y$ from some special Kummer K3 surface $X$ in odd characteristic. In Section 3, we define this surface $X$ and prove Theorem 1.1 (2) by studying the surface $X$ and its suitable blow-up. Complex surfaces similar to $X$ are fully studied in Og89 and effectively applied in [DO19]. However, some arguments there are based on the global Torelli theorem for complex K3 surfaces (see eg. [BHPV04, Chapter VIII]) which is not available over $k$. We also use a result due to Jang [Ja13, Proposition 3.5] on the finiteness of canonical representation of any non-supersingular K3 surface defined over any algebraically closed field of odd characteristic (Theorem 3.7). This substitutes the finiteness of canonical representation in characteristic 0 ([Ue75, Theorem 14.10]) used in [DO19].

We prove Theorem 1.1 (2) in Section 3 and Corollary 1.2 in Section 4 .

Throughout this paper, for a variety $V$ defined over a field $K$ and for closed subsets $W_{1}$, $W_{2}, \ldots, W_{n}$ of $V$, we denote

$$
\operatorname{Aut}\left(V / K, W_{1}, W_{2}, \ldots, W_{n}\right):=\left\{f \in \operatorname{Aut}(V / K) \mid f\left(W_{i}\right)=W_{i}(\forall i)\right\} .
$$

Acknowledgements. I would like to thank Professors Tien-Cuong Dinh, Igor Dolgachev, Jun-Muk Hwang, Hélène Esnault, Yuya Matsumoto, Junichiro Noguchi, Takeshi Saito for valuable discussion and help. Especially, I would like to express my thanks to Professor Tien-Cuong Dinh for sharing many ideas since our previous joint work [DO19] and his warm encouragement, Professor Jun-Muk Hwang for his invitation to one day workshop at KIAS which was very helpful to make the final version of this paper and Professor Hélène Esnault and the referee for many valuable comments most of which are effectively reflected in this paper.

\section{Proof of Theorem 1.1 (1)}

Our main result of this section is Theorem 2.4 (3). We then deduce Theorem 1.1(1) as an application of Theorem 2.4 (3) and Lemma 2.2 below.

As in [DO19], the following theorem will be frequently used in this paper.

Theorem 2.1. Let $G$ be a group and $H \subset G$ a subgroup of $G$. Assume that $H$ is of finite index, i.e., $[G: H]<\infty$. Then, the group $H$ is finitely generated if and only if $G$ is finitely generated.

Proof. "Only if part" is clear. "If part" follows from a standard method finding a set of generators of $H$ from a given set of generators of $G$ and complete representatives of the left coset $G / H$, called Reidemeister's method. See e.g. [Su82, Page 181, Corollary 1] for a self-contained proof.

The following lemma is implicitly used in several papers. Our argument here is much inspired by a paper of Professor János Kollár [Ko09, Proof of Theorem 6]:

Lemma 2.2. Let $K$ be an algebraically closed field and let $V$ be a projective variety defined over $K$. Assume that Aut $(V / K)$ is discrete. Then, Aut $(V / K)=\operatorname{Aut}\left(V_{L} / L\right)$, as groups, for any field extension $K \subset L$, under the natural inclusion $\operatorname{Aut}(V / K) \subset \operatorname{Aut}\left(V_{L} / L\right)$. 
Proof. Let $\varphi \in \operatorname{Aut}\left(V_{L} / L\right) \backslash \operatorname{Aut}(V / K)$. Since $K$ is algebraicaly closed and $\varphi \notin \operatorname{Aut}(V / K)$, the residue field of the point

$$
[\varphi]: \operatorname{Spec} L \rightarrow \operatorname{Aut}\left(X_{L} / L\right) \subset \operatorname{Hilb}\left(V_{L} \times V_{L}\right)
$$

corresponding to the graph of $\varphi$ is transcendental over $K$. However, then, the specialization gives a positive dimensional subset of Aut $(V / K) \subset \operatorname{Hilb}(V \times V)$, a contradiction to our assumption that Aut $(V / K)$ is discrete. This implies the result.

Remark 2.3. Needless to say, Aut $\left(V_{L} / L\right)$ is much bigger than Aut $(V / K)$ in general. For instance, for an elliptic curve $E$ defined over $\overline{\mathbb{Q}}$, the group $\operatorname{Aut}\left(E_{\mathbb{C}} / \mathbb{C}\right)$ is uncountable, while $\operatorname{Aut}(E / \overline{\mathbb{Q}})$ is countable.

We denote by $\kappa(X)$ the Kodaira dimension of a smooth projective variety $X$.

Theorem 2.4. Let $K$ be an algebraically closed field.

(1) Let $X$ be a variety defined over $K$. We assume that $X$ is a smooth projective surface such that either $\kappa(X) \geq 1$ or the image of the albanese morphism $X \rightarrow \operatorname{Alb}(X)$ is a curve, or $X$ is an abelian variety. Then the group $\operatorname{Aut}(X / K) / \operatorname{Aut}^{0}(X / K)$ is finitely generated. In particular, the automorphism group $\operatorname{Aut}_{\text {group }}(X / K)$ of an abelian variety $X$ as a group variety is finitely generated.

(2) Let $X$ be a smooth minimal projective surface defined over $K$. Assume that $\kappa(X)=$ 0 and $K$ is of odd characteristic. Then the group $\operatorname{Aut}(X / K) / \operatorname{Aut}^{0}(X / K)$ is finitely generated.

(3) Let $Y$ be a smooth projective surface defined over $k_{0}$, an algebraic closure of the prime field $\mathbb{F}_{p}$ of odd characteristic. Then, the group $\operatorname{Aut}\left(Y / k_{0}\right) / \operatorname{Aut}^{0}\left(Y / k_{0}\right)$ is finitely generated unless $Y$ is a rational surface.

Proof. The assertion (1) should be known for the experts. We give a proof for the convenience of the readers. Let

$$
\tau: \operatorname{Aut}(X / K) / \operatorname{Aut}^{0}(X / K) \rightarrow O(\mathrm{NS}(X)) /(\text { torsion }) ;\left.f \mapsto f^{*}\right|_{\mathrm{NS}(X) /(\text { torsion })}
$$

be the natural contravariant group homomorphism. Then $\operatorname{Ker}(\tau)$ is a finite group by $[\operatorname{Br} 19$, Theorem 2.10]. Thus, it suffices to show that the group $\operatorname{Im}(\tau)$ is finitely generated.

First consider the case where $X$ is a surface.

Assume that $\kappa(X) \geq 1$. Let $m K_{X}=P+N$ be the Zariski decomposition of $m K_{X}$ where $m$ is a sufficiently divisible positive integer. Then $P \in \mathrm{NS}(X) /$ (torsion). We have $\left(P^{2}\right)>0$ when $\kappa(X)=2$ and $P \neq 0$ and $\left(P^{2}\right)=0$ when $\kappa(X)=1$. Since the class $P$ is preserved by $\operatorname{Im}(\tau)$, it follows from the Hodge index theorem that $\operatorname{Im}(\tau)$ is finite when $\kappa(X)=2$ and $\operatorname{Im}(\tau)$ is a finitely generated abelian group up to finite kernel and cokernel when $\kappa(X)=1$ (See eg. Og07, Theorem 2.1]). Hence the group $\operatorname{Im}(\tau)$ is finitely generated.

Assume that the image of the albanese morphism $X \rightarrow \operatorname{Alb}(X)$ is a curve. Then the class of general fiber $F \in \mathrm{NS}(S) /$ (torsion) satisfies $F \neq 0$ and $\left(F^{2}\right)=0$ and is preserved by $\operatorname{Im}(\tau)$. Hence for the same reason as in $\kappa(X)=1$, the group $\operatorname{Im}(\tau)$ is a finitely generated abelian group up to finite kernel and cokernel, in particular, finitely generated.

Next, consider the case where $X$ is an abelian variety. Let $O$ be the origin of the group $X$. Then $\operatorname{Aut}(X / K) / \operatorname{Aut}^{0}(X / K)$ is isomorphic to $\operatorname{Aut}(X / K, O)=\operatorname{Aut}_{\text {group }}(X / K)$, which is an arithmetic subgroup of the real linear algebraic group $\left(\operatorname{End}^{0}(X) \otimes \mathbb{R}\right)^{\times}$defined over $\mathbb{Q}$ (See eg. [PS12, Corollary 3.6]). Hence the group $\operatorname{Aut}(X / K) / \operatorname{Aut}^{0}(X / K)$ is finitely generated by [BH62, Theorem 6.2]. 
This completes the proof of the assertion (1).

Let us show the assertion (2). By (1) and by the classification of smooth projective surfaces ([BM77]), we may assume that $X$ is either a K3 surface or an Enriques surface. Since $K$ is of odd characteristic, the result follows from Theorem 1.4 (the main result of [LM18]) for K3 surfaces and [Wa19, Theorem 1.3] for Enriques surfaces. This completes the proof of the assertion (2).

We show the assertion (3). Note that $Y$ is not a rational surface by our assumption. Then, by (1) and (2) and by the classification of smooth projective surfaces ([BM77]), we may assume that $Y$ is not minimal and $Y$ is birational to either a $\mathrm{K} 3$ surface, an Enriques surface, or an abelian surface.

Let $X$ be the minimal model of $Y$. Then the surface $X$ is unique up to isomorphism and we have a birational morphism

$$
\pi=\pi_{n} \circ \pi_{n-1} \circ \ldots \circ \pi_{0}: Y:=X_{n+1} \rightarrow X_{n} \rightarrow \ldots \rightarrow X_{1} \rightarrow X_{0}:=X,
$$

where $\pi_{i}: X_{i+1} \rightarrow X_{i}$ is the blow-up at some point $P_{i} \in X_{i}\left(k_{0}\right)$. For the same reason as in Remark $1.3(2)$, Aut $\left(Y / k_{0}\right) \subset \operatorname{Aut}\left(X / k_{0}\right)$ via $\pi$. Let $E_{\pi} \subset Y$ be the exceptional set of $\pi$. Since $Y$ is not minimal, $\pi\left(E_{\pi}\right)$ is a non-empty finite set of points, hence $P_{0} \in \pi\left(E_{\pi}\right)$, and the group Aut $\left(Y / k_{0}\right)$ preserves $\pi\left(E_{\pi}\right)$ via $\pi$. Then,

$$
H:=\operatorname{Aut}\left(Y / k_{0}, \pi^{-1}\left(P_{0}\right)\right)
$$

is a finite index subgroup of Aut $\left(Y / k_{0}\right)$ such that $H \subset \operatorname{Aut}\left(X / k_{0}, P_{0}\right)$ via $\pi$.

We are going to show that $H$ is finitely generated. First we observe the following:

Lemma 2.5. Aut $\left(X / k_{0}, P_{0}\right)$ is a finitely generated group.

Proof. The result follows from Theorem 2.4 (1) when $X$ is an abelian surface. Indeed, we may take $P_{0}$ as the origin of $X$.

Assume that $X$ is a $\mathrm{K} 3$ surface or an Enriques surface. Then Aut $\left(X / k_{0}\right)$ is finitely generated by Theorem 2.4 (2). We set

$$
\operatorname{Aut}\left(X / k_{0}\right)=\left\langle h_{1}, \ldots, h_{r}\right\rangle \text {. }
$$

Then there is a positive integer $q$, which is a power of $p$, such that $h_{j}(1 \leq j \leq r)$ are all defined over $\mathbb{F}_{q}$ and also $P_{0} \in X\left(\mathbb{F}_{q}\right)$. By definition, any $h \in \operatorname{Aut}\left(X / k_{0}\right)$ is then defined over $\mathbb{F}_{q}$. Let $T=X\left(\mathbb{F}_{q}\right)$. Then $T$ is a finite set. Since $T$ is preserved by each $h_{j}$, it follows that $T$ is preserved by $\operatorname{Aut}\left(X / k_{0}\right)$. Hence we have a group homomorphism

$$
\sigma: \operatorname{Aut}\left(X / k_{0}\right) \rightarrow \operatorname{Aut}_{\text {set }}(T)
$$

and

$$
\operatorname{Ker}(\sigma) \subset \operatorname{Aut}\left(X / k_{0}, P_{0}\right) \subset \operatorname{Aut}\left(X / k_{0}\right) .
$$

Then $\operatorname{Ker}(\sigma)$ is a finite index subgroup of Aut $\left(X / k_{0}\right)$ by $|T|<\infty$. Hence Aut $\left(X / k_{0}, P_{0}\right)$ is a finite index subgroup of Aut $\left(X / k_{0}\right)$ as well. Since Aut $\left(X / k_{0}\right)$ is finitely generated, so is $\operatorname{Aut}\left(X / k_{0}, P_{0}\right)$ by Theorem 2.1. This completes the proof of the assertion (3).

By Lemma 2.5, we may set

$$
\operatorname{Aut}\left(X / k_{0}, P_{0}\right)=\left\langle g_{1}, \ldots, g_{m}\right\rangle .
$$

Then there is a positive integer $q$, which is a power of $p$, such that $g_{j}$ are all defined over $\mathbb{F}_{q}$ and also $P_{i} \in X_{i}\left(\mathbb{F}_{q}\right)$ for all integers $0 \leq i \leq n+1$ and $1 \leq j \leq m$. By definition, any $g \in \operatorname{Aut}\left(X / k_{0}, P_{0}\right)$ is then defined over $\mathbb{F}_{q}$ and the blow-up $\pi_{i}$ are also defined over $\mathbb{F}_{q}$. 
Let $S=X\left(\mathbb{F}_{q}\right)$. Then $S$ is a finite set. We consider the blow-up $p_{0}: Y_{1} \rightarrow Y_{0}:=X$ at $S$ and the exceptional divisor $E_{S}$ of $p_{0}$. Here $E_{S}$ is a disjoint union of $|S| \mathbb{P}^{1}$ s. Then $Y_{1}$ is defined over $\mathbb{F}_{q}$ and $S_{1}:=E_{S}\left(\mathbb{F}_{q}\right)$ is a finite set. We then consider the blow-up $p_{1}: Y_{2} \rightarrow Y_{1}$ at $S_{1}$. Then $Y_{2}$ is defined over $\mathbb{F}_{q}$. We repeat this process $(n+1)$-times, where $n$ is the same positive integer $n$ as in $\pi: Y \rightarrow X$ above, and get the birational morphism

$$
\varphi:=p_{n} \circ p_{n-1} \circ \ldots \circ p_{0}: Z:=Y_{n+1} \rightarrow Y_{n} \rightarrow \ldots \rightarrow Y_{1} \rightarrow Y_{0}=X .
$$

By the choice of $\mathbb{F}_{q}$ and by the construction of $Z$, each element of Aut $\left(X / k_{0}, P_{0}\right)$ lifts to an element of $\operatorname{Aut}\left(Z / k_{0}, \varphi^{-1}\left(P_{0}\right)\right)$ under $\varphi$. Thus the inclusion

$$
\operatorname{Aut}\left(Z / k_{0}, \varphi^{-1}\left(P_{0}\right)\right) \subset \operatorname{Aut}\left(X / k_{0}, P_{0}\right)
$$

induced by $\operatorname{Aut}\left(Z / k_{0}\right) \subset \operatorname{Aut}\left(X / k_{0}\right)$ via $\varphi$ is actually an equality, that is,

$$
\left.\operatorname{Aut}\left(Z / k_{0}, \varphi^{-1}\left(P_{0}\right)\right)\right)=\operatorname{Aut}\left(X / k_{0}, P_{0}\right)
$$

via $\varphi$. Let $\left\{E_{j}\right\}_{j=1}^{N}$ be the set of the irreducible components of the exceptional divisor of $\varphi$. By construction, the set $\left\{E_{j}\right\}_{j=1}^{N}$ is preserved by Aut $\left(X / k_{0}, P_{0}\right)$ under the identification made above. Thus, we have a group homomorphism

$$
\rho: \operatorname{Aut}\left(X / k_{0}, P_{0}\right) \rightarrow \operatorname{Aut}_{\text {set }}\left(\left\{E_{i}\right\}_{i=1}^{N}\right) \simeq S_{N}
$$

Here $S_{N}$ is the symmetric group of $N$ letters. Let $G=\operatorname{Ker}(\rho)$. Then

$$
\left[\operatorname{Aut}\left(X / k_{0}\right): G\right]=|\operatorname{Im} \rho| \leq\left|S_{N}\right|=N !<\infty \text {. }
$$

On the other hand, again by our choice of $\mathbb{F}_{q}$ and the construction of $Z$, we have the factorization $\tau: Z \rightarrow Y$ of $\varphi: Z \rightarrow X$ by $\pi: Y \rightarrow X:$

$$
\varphi=\pi \circ \tau: Z \rightarrow Y \rightarrow X
$$

Then $\tau$ is the smooth blow-down of some irreducible curves in $\left\{E_{j}\right\}_{j=1}^{N}$. By the definition, $G$ preserves each element $E_{j}$ of $\left\{E_{j}\right\}_{j=1}^{N}$. Thus any element of $G$ descends to $H=\operatorname{Aut}\left(Y, \pi^{-1}\left(P_{0}\right)\right)$ via $\tau$. Hence we have the following group inclusions

$$
G \subset H \subset \operatorname{Aut}\left(X / k_{0}, P_{0}\right)
$$

via $\tau$ and $\pi$. The resulting inclusion $G \subset \operatorname{Aut}\left(X / k_{0}, P_{0}\right)$ is then the same as the one via $\varphi=\pi \circ \tau$. Thus

$$
\left[\operatorname{Aut}\left(X / k_{0}, P_{0}\right): H\right] \leq\left[\operatorname{Aut}\left(X / k_{0}, P_{0}\right): G\right]<\infty .
$$

Recall that $\operatorname{Aut}\left(X / k_{0}, P_{0}\right)$ is finitely generated (Lemma 2.5). Hence by Theorem 2.1, $H$ is finitely generated. Since $H$ is a finite index subgroup of $\operatorname{Aut}\left(Y / k_{0}\right)$, the group $\operatorname{Aut}\left(Y / k_{0}\right)$ is also finitely generated as well by Theorem 2.1. This completes the proof of the assertion (3).

We are ready to prove Theorem 1.1 (1). We use the same notation as in Theorem 1.1 (1). Since $Y$ is birational to a K3 surface, Aut $\left(Y / k_{0}\right)$ is discrete (Remark $1.3(2)$ ). Thus, by Theorem $2.4(3)$, Aut $\left(Y / k_{0}\right)$ is a finitely generated group. Hence Aut $\left(Y_{L} / L\right)$ is finitely generated as well by Lemma 2.2. This completes the proof of Theorem 1.1 (1). 


\section{Proof of Theorem 1.1 (2)}

In this section, we prove Theorem 1.1(2) by constructing $Y$ explicitly from an explicitly given Kummer K3 surface $X$ below. Our main result of this section is Theorem 3.9. As mentioned in the introduction, our construction is very close to the one in [DO19].

Let $k$ be an algebraically closed field as in Introduction. Recall that

$$
t \in \mathbb{F}_{p}(t) \subset k
$$

and $t$ is transcendental over $\mathbb{F}_{p}$.

We finally reduce our proof of non-finite generation to the following lemma.

Lemma 3.1. The subgroup $G_{t}:=\left\langle t^{n} \mid n \in \mathbb{Z}\right\rangle$ of the additive group $k=(k,+)$ is not finitely generated.

Proof. If otherwise, $G_{t}$ would be a finitely generated abelian group with $\mathbb{F}_{p}$-vector space structure induced by the one on $k$. So $G$ has to be a finite dimensional $\mathbb{F}_{p}$-vector space, say of dimension $d$. Then the following $d+1$ elements

$$
1, t, t^{2}, \ldots, t^{d}
$$

of $G_{t}$ has to be linearly dependent over $\mathbb{F}_{p}$. Thus, there is

$$
(0,0, \ldots, 0) \neq\left(a_{0}, a_{1}, \ldots, a_{d}\right) \in \mathbb{F}_{p}^{\oplus d}
$$

such that

$$
a_{0}+a_{1} t+\ldots+a_{d} t^{d}=0
$$

in $G_{t} \subset k$. However, this contradicts to the fact that $t$ is transcendental over $\mathbb{F}_{p}$.

Let $E$ be the elliptic curve defined over $k$ by the Weierstrass equation

$$
y^{2}=x(x-1)(x-t) .
$$

Note that $E /\left\langle-1_{E}\right\rangle=\mathbb{P}^{1}$, the associated quotient map $E \rightarrow \mathbb{P}^{1}$ is given by $(x, y) \mapsto x$ and the points $0,1, t$ and $\infty$ of $\mathbb{P}^{1}(k)$ are exactly the branch points of this quotient map.

Let $F$ be any elliptic curve defined over $k$ such that $F$ is not isogenous to $E$. For instance, we may take a supersingular elliptic curve defined over $k$ as $F$. Note that there certainly exists a supersingular elliptic curve $F$ over $k$ and $E$ is not a supersingular (see eg. subsection "Elliptic curves in Characteristic $p>0$ " in [Mu74, Section 22]). In particular, $E$ and $F$ are not isogenous over $k$ (see eg. subsection "The $p$-rank" in [Mu74, Section 15]).

Throughout this section, we denote by

$$
X:=\operatorname{Km}(E \times F)
$$

the Kummer K3 surface accociated to the product abelian surface $E \times F$, that is, the minimal resolution of the quotient surface $E \times F /\left\langle\left(-1_{E},-1_{F}\right)\right\rangle$. We write $H^{0}\left(X, \Omega_{X}^{2}\right)=$ $k \omega_{X}$. Then $\omega_{X}$ is a nowhere vanishing regular global 2-form on $X$ and it is induced by a nowhere vanishing regular global 2-form on $E \times F$.

Since $E$ and $F$ are not isogenous, the Picard number $\rho(E \times F)$ of $E \times F$ is 2 and therefore the Picard number $\rho(X)$ of $X$ is 18 by [Sh75, Proposition 1 and Appendix]. In particular, our K3 surface $X$ is not supersingular.

Let $\left\{a_{i}\right\}_{i=1}^{4}$ and $\left\{b_{i}\right\}_{i=1}^{4}$ be the 2-torsion subgroups of $F$ and $E$ respectively. Then $X$ contains 24 "visible" smooth rational curves as in Figure 1. Here smooth rational curves $E_{i}, F_{i}(1 \leq i \leq 4)$ are arising from the elliptic curves $E \times\left\{a_{i}\right\},\left\{b_{i}\right\} \times F$ on $E \times F$. Smooth 


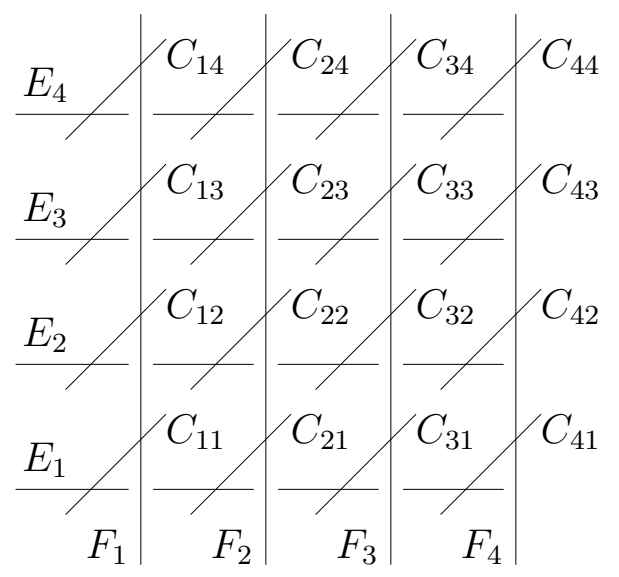

Figure 1. Curves $E_{i}, F_{j}$ and $C_{i j}$

rational curves $C_{i j}(1 \leq i, j \leq 4)$ are the exceptional curves over the $A_{1}$-singular points of the quotient surface $E \times F /\left\langle-1_{E \times F}\right\rangle$. Throughout this section, we will freely use the names of curves in Figure 1 .

Definition 3.2. As in [DO19], we set

$$
C:=E_{1}=E /\left\langle-1_{E}\right\rangle \subset X .
$$

We may and do use $x$ in the Weierstrass equation of $E$ as an affine coordinate on $C$ and also assume that under the affine coordinate $x$,

$$
C \cap C_{11}=\{\infty\}, C \cap C_{21}=\{0\}, C \cap C_{31}=\{1\}, C \cap C_{41}=\{t\} .
$$

We define the point $P \in C(k) \subset X(k)$ by

$$
P:=\infty,
$$

that is, the intersection point of $C$ and $C_{11}$.

Let

$$
\theta=\left[\left(1_{E},-1_{F}\right)\right]=\left[\left(-1_{E}, 1_{F}\right)\right] \in \operatorname{Aut}(X / k)
$$

be the automorphism of $X$ induced by the automorphism $\left(1_{E},-1_{F}\right) \in \operatorname{Aut}((E \times F) / k)$ of $E \times F$. Then $\theta$ is of order 2 . Set

$$
B:=\cup_{i=1}^{4} E_{i} \cup \cup_{j=1}^{4} F_{j} .
$$

The following theorem was proved in Og89, Lemmas (1.3), (1.4)] over $\mathbb{C}$. However, the proof there is based on the global Torelli theorem for complex K3 surfaces and Hodge theory. So, one can not apply the argument there for our $X$ over $k$.

Theorem 3.3. The following properties hold also over $k$.

(1) The Picard group Pic $(X)$ is torsion free.

(2) $\theta^{*}=\mathrm{id}$ on $\operatorname{Pic}(X)$ and $\theta^{*} \omega_{X}=-\omega_{X}$.

(3) Aut $((E \times F) / k)=\operatorname{Aut}(E / k) \times \operatorname{Aut}(F / k)$ and $f \circ \theta=\theta \circ f$ for all $f \in \operatorname{Aut}(X / k)$.

(4) Let $X^{\theta}$ be the fixed locus of $\theta$. Then $X^{\theta}=B$.

(5) $\operatorname{Aut}(X / k)=\operatorname{Aut}(X / k, B)$. 
Proof. Assume that $L \in \operatorname{Pic}(X)$ satisfies $n L=0$ in $\operatorname{Pic}(X)$ for some positive integer $n$. Then $(L, L)_{X}=0$ and therefore $\chi(X, L)=2$ by the Riemann-Roch formula. Combining this with the Serre duality, we deduce that either $L$ or $-L$ is represented by an effective divisor. This implies $L=0$, as $( \pm L, H)_{X}=0$ for a very ample divisor $H$ on $X$ by $n L=0$ $(n \neq 0)$. This proves $(1)$.

By $\rho(X)=18$, we see that $\operatorname{Pic}(X) \otimes \mathbb{Q}$ is generated by the 24 rational curves in Figure 1. It is clear that $\theta$ preserves each of these 24 curves. It follows that $\theta^{*}=\mathrm{id}$ on $\operatorname{Pic}(X) \otimes \mathbb{Q}$ and therefore $\theta^{*}=\mathrm{id}$ also on Pic $(X)$ by (1). By the shape of $\theta$, clearly $\theta^{*} \omega_{X}=-\omega_{X}$. This shows (2).

The first assertion of (3) is an immediadte consequence of our assumption that $E$ and $F$ are not isogenous. Let

$$
g:=\theta \circ f \circ \theta^{-1} \circ f^{-1} \in \operatorname{Aut}(X / k) .
$$

We are going to show that $g=\mathrm{id}_{X}$. We have $g^{*}=i d$ on Pic $(X)$ by $(1)$ and $g^{*} \omega_{X}=\omega_{X}$ by the definition of $g$. In particular, $g(R)=R$ for all smooth rational curves $R \subset X$. This is because $g^{*}(R)=R$ in $\operatorname{Pic}(X)$ and $|R|=\{R\}$ by $\left(R^{2}\right)=-2$. Here we recall that $\left(R^{2}\right)=-2$ for any smooth rational curve $R$ on $X$ by the adjunction formula. Thus

$$
g\left(\sum_{i, j} C_{i j}\right)=\sum_{i, j} C_{i j}
$$

Let $V$ be the blow-up of $E \times F$ at the sixteen 2-torsion points $P_{i j}$ and $D_{i j} \subset V$ the exceptional curve over the 2-torsion point $P_{i j}$. The induced morphism $\pi: V \rightarrow X$ is a finite cover of degree 2 branched along $\sum_{i, j} C_{i j}$. Since Pic $(X)$ is torsion free and since any degree 2 map is separable over $k$ of odd characteristic, the converse is also true. That is, if $\pi^{\prime}: V^{\prime} \rightarrow X$ is a finite double cover branched along $\sum_{i, j} C_{i j}$, then $\pi: V \rightarrow X$ and $\pi^{\prime}: V^{\prime} \rightarrow X$ are isomorphic over $X$ (See eg. [Fu83, Theorem 2.6]). Applying this for $g \circ \pi: V \rightarrow X$ and $\pi: V \rightarrow X$, we deduce that $g$ lifts to an automorphism $g_{V}$ of $V$ such that $g_{V}\left(D_{i j}\right)=D_{i j}$ for each $(i, j)$. Then $g_{V}$ descends to the automorphism $g_{E \times F}$ of $E \times F$ such that

$$
g_{E \times F}\left(P_{i j}\right)=P_{i j}
$$

for each 2-torsion point $P_{i j}$ of $E \times F$ and

$$
g_{E \times F}^{*} \omega_{E \times F}=\omega_{E \times F} .
$$

Write $g_{E \times F}=\left(g_{E}, g_{F}\right)\left(g_{E} \in \operatorname{Aut}(E / k), g_{F} \in \operatorname{Aut}(F / k)\right)$ by using the first assertion of (3). Then $g_{E}= \pm \operatorname{id}_{E}$ and $g_{F}= \pm \operatorname{id}_{F}$ by $g_{E \times F}\left(P_{i j}\right)=P_{i j}$. Combining this with $g_{E \times F}^{*} \omega_{E \times F}=\omega_{E \times F}$, we obtain that $g_{E \times F}= \pm \operatorname{id}_{E \times F}$. Hence $g=\operatorname{id}_{X}$, i.e., $\theta \circ f=f \circ \theta$ on $X$. This proves $(3)$.

The assertion (4) is immediate from the shape of $\theta$. The assertion (5) follows from (3) and (4). This completes the proof.

Proposition 3.4. Aut $(X / k, P) \subset \operatorname{Aut}(X / k, C)$. That is, $f(C)=C$ holds for every $f \in \operatorname{Aut}(X / k, P)$. In particular,

$$
\operatorname{Aut}(X / k, P)=\operatorname{Aut}(X / k, C, P) \text {. }
$$

Proof. By Theorem 3.3, we have Aut $(X)=\operatorname{Aut}(X, B)$. This implies the result, because $C$ is the unique irreducible component of $B$ such that $P \in C(k)$.

Lemma 3.5. Let $R \subset X$ be a smooth rational curve such that $R \not \subset B$. Then 
(1) $\theta(R)=R$ and $\left.\theta\right|_{R} \in$ Aut $(R / k)$ is of order 2. Moreover, $\left.\theta\right|_{R}$ has exactly two fixed closed points and $d\left(\left.\theta\right|_{R}\right)_{Q}=-1$ at each fixed closed point $Q \in R(k)$ of $\left.\theta\right|_{R}$.

(2) Assume furthermore that $P \in R(k)$. Then, for each $f \in \operatorname{Aut}(X / k, P)$, either $f(R)=R$ or $f(R)$ and $R$ are tangent at $P$.

Proof. Note that $-1 \neq 1$ in the field $k$ of odd characteristic. So, once Theorem 3.3 is established, then exactly the same proof as [DO19, Lemma 3.5] works also over $k$.

Recall that Aut $(X / k, P)=\operatorname{Aut}(X / k, C, P)$ (Proposition 3.4). We define two differential representations of Aut $(X / k, P), d_{X, P}$ on the tangent space $T_{X, P} \simeq k^{2}$ and $d_{X, C, P}$ on the tangent space $T_{C, P} \simeq k$, and two subgroups $G(X, P)$ and $G(X, C, P)$ of Aut $(X / k, P)$ by

$$
\begin{gathered}
d_{X, P}: \operatorname{Aut}(X / k, P) \rightarrow \mathrm{GL}\left(T_{X, P}\right) ; f \mapsto d f_{P}, \\
d_{X, C, P}: \operatorname{Aut}(X / k, P) \rightarrow \mathrm{GL}\left(T_{C, P}\right) ; f \mapsto d\left(\left.f\right|_{C}\right)_{P}, \\
G(X, P):=\operatorname{Ker}\left(d_{X, P}: \operatorname{Aut}(X / k, P) \rightarrow \operatorname{GL}\left(T_{X, P}\right) ; f \mapsto d f_{P}\right), \\
G(X, C, P):=\operatorname{Ker}\left(d_{X, C, P}: \operatorname{Aut}(X / k, P) \rightarrow \operatorname{GL}\left(T_{C, P}\right) ; f \mapsto d\left(\left.f\right|_{C}\right)_{P}\right) .
\end{gathered}
$$

Clearly $G(X, P) \subset G(X, C, P)$ as groups.

Let $0 \neq v_{1} \in T_{C, P} \subset T_{X, P}$ and $0 \neq v_{2} \in T_{C_{11}, P} \subset T_{X, P}$. Then $\left\langle v_{1}, v_{2}\right\rangle$ forms a basis of the $k$-vector space $T_{X, P}$.

Proposition 3.6. $\operatorname{Im}\left(d_{X, P}\right)$ is simultaneously diagonalizable with respect to the basis $\left\langle v_{1}, v_{2}\right\rangle$ of $T_{X, P}$.

Proof. This is because $d f_{P}(f \in \operatorname{Aut}(X / k, P))$ preserves $T_{C, P}$ and also preserves $T_{C_{11}, P}$ by Lemma 3.5(2).

Let $K$ be any algebraically closed field of odd characteristic and let $S$ be any K3 surface defined over $K$. Then we have $H^{0}\left(S, \Omega_{S}^{2}\right)=K \omega_{S} \simeq K$ and for each $f \in \operatorname{Aut}(S / K)$, there is a unique $\alpha(f) \in K^{\times}$such that $f^{*} \omega_{S}=\alpha(f) \omega_{S}$. The group homomorphism

$$
\alpha: \operatorname{Aut}(S / K) \rightarrow \mathrm{GL}\left(K \omega_{S}\right)=K^{\times} ; f \mapsto \alpha(f)
$$

is called the canonical representation of $S$ or of $\operatorname{Aut}(S / K)$.

Theorem 3.7. The image $\alpha$ (Aut $(S / K))$ of the canonical representation is a finite group, hence a finite cyclic group, for any non-supersingular K3 surface $S$ defined over any algebraically closed field $K$ of odd characteristic.

Proof. This is proved by Jang [Ja13, Proposition 3.5] as an important application of Theorem 1.4 (2) due to Lieblich and Maulik. Here we recall the proof for the convenience of the readers. Let $\pi: X \rightarrow$ Spec $R$ be the lifting of $S$ in Theorem 1.4 (2). Let $\omega_{X / R}$ be the relative regular 2-form of $\pi$. Consider the canonical representation $\alpha_{S}=\alpha$ of $S$ :

$$
\alpha_{S}: \operatorname{Aut}(S / K) \rightarrow \operatorname{GL}\left(\left.K \omega_{X / R}\right|_{S}\right)=K^{\times} .
$$

Let $G$ be the image of the specialization map

$$
\operatorname{Aut}(\tilde{S} / \tilde{K}) \rightarrow \operatorname{Aut}(S / K)
$$

in Theorem 1.4 (2). Let $\mathbf{m}$ be the maximal ideal of $R$. Then the homomorphism

$$
\left.\alpha_{S}\right|_{G}: G \rightarrow \mathrm{GL}\left(\left.K \omega_{X / R}\right|_{S}\right)=K^{\times}
$$


is the mod $\mathbf{m}$-reduction of the canonical representation of $\tilde{S}$ :

$$
\alpha_{\tilde{S}}: \operatorname{Aut}(\tilde{S} / \tilde{K}) \rightarrow \operatorname{GL}\left(\left.\tilde{K} \omega_{X / R}\right|_{\tilde{S}}\right)=\tilde{K}^{\times} .
$$

Since $\tilde{K}$ is of characteristic 0 , the group $\operatorname{Im} \alpha_{\tilde{S}}$ is a finite cyclic group by Ue75, Theorem 14.10]. Therefore $\left.\operatorname{Im} \alpha_{S}\right|_{G}=\alpha_{S}(G)$ is also a finite cyclic group. Since [Aut $\left.(S / K): G\right]<\infty$ by Theorem $2.1(2)$, it follows that $\alpha_{S}($ Aut $(S / K))$ is a finite subgroup of $K^{\times}$. Hence it is a finite cyclic group as claimed.

Recall that $G(X, P)$ is a subgroup of $G(X, C, P)$.

Proposition 3.8. (1) $[G(X, C, P): G(X, P)]<\infty$.

(2) $G(X, P)$ is not finitely generated.

Proof. First, we prove the assertion (1). Let $f \in \operatorname{Aut}(X / k, P)$. Then by Proposition 3.6, we have

$$
d f_{P}\left(v_{1}\right)=\alpha_{1}(f) v_{1}, d f_{P}\left(v_{2}\right)=\alpha_{2}(f) v_{2}
$$

for some $\alpha_{1}(f), \alpha_{2}(f) \in k^{\times}$. Then for the canonical representation $\alpha$ of Aut $(X / k)$, we have

$$
\alpha(f)=\alpha_{1}(f) \alpha_{2}(f) .
$$

Then $\alpha(f)=\alpha_{2}(f)$ for $f \in G(X, C, P)$, as $\alpha_{1}(f)=1$ for $f \in G(X, C, P)$. Thus

$$
G(X, P)=\operatorname{Ker}\left(\left.\alpha\right|_{G(X, C, P)}\right)
$$

by Proposition [3.6, and therefore

$$
[G(X, C, P): G(X, P)]=\left|\operatorname{Im}\left(\left.\alpha\right|_{G(X, C, P)}\right)\right| \leq|\operatorname{Im}(\alpha)|<\infty,
$$

by Theorem 3.7. This completes the proof of the assertion (1).

Next we shall prove the assertion (2). Consider the group representation

$$
\tau: G(X, C, P) \rightarrow \operatorname{Aut}(C, P) ;\left.f \mapsto f\right|_{C} .
$$

Let

$$
\Gamma:=\tau(G(X, C, P)), f \in G(X, C, P) .
$$

Then by the definition of $G(X, C, P)$, we have $\left.f\right|_{C}(P)=P$ and $d\left(\left.f\right|_{C}\right)_{P}=1$ on $C=\mathbb{P}^{1}$. Under the affine coordinate $x$ of $C$, the automorphism $\left.f\right|_{C} \in \operatorname{Aut}(C / k)$ is then of the form

$$
f(x)=x+a_{f} \quad \text { with } \quad a_{f} \in k .
$$

Thus $\Gamma$ is isomorphic to a subgroup of the additive group $k=(k,+)$ and therefore $\Gamma$ is an abelian group with $\mathbb{F}_{p}$-linear space structure.

Now, to conclude Proposition 3.8 (2), it suffices to show that $\Gamma$ do have a non-finitely generated subgroup. Indeed, then, $\Gamma$ is not also finitely generated, as $\Gamma$ is an abelian group. Hence $G(X, C, P)$ is not finitely generated, as its image $\Gamma:=\tau(G(X, C, P))$ is not finitely generated. Since $[G(X, C, P): G(X, P)]<\infty$ by Proposition $3.8, G(X, P)$ is not finitely generated as well, by Theorem 2.1 .

In the rest, we will find a non-finitely generated subgroup of $\Gamma$ by constructing various (quasi-)elliptic fibrations with section on $X$.

As in DO19, consider the following two divisors $D_{1}$ and $D_{2}$ of Kodaira's type $I_{8}$ and $I V^{*}$ on $X$ :

$$
\begin{gathered}
D_{1}:=C+C_{11}+F_{1}+C_{12}+E_{2}+C_{22}+F_{2}+C_{21}, \\
D_{2}:=C+2 C_{11}+E_{2}+2 C_{12}+E_{3}+2 C_{13}+3 F_{1} .
\end{gathered}
$$


Observe also that

$$
\left(D_{1} \cdot C_{31}\right)=\left(D_{1} \cdot C_{41}\right)=1,\left(D_{2} \cdot C_{21}\right)=\left(D_{2} \cdot C_{31}\right)=1 \text {. }
$$

Thus, by [DO19, Prop. 3.8], which is also valid over any algebraically closed field $K$ (if one replaces the term "elliptic" there by "quasi-elliptic" when $K$ is of characteristic 2,3 ), we obtain two (quasi-)elliptic fibrations

$$
\varphi_{D_{1}}: X \rightarrow \mathbb{P}^{1}
$$

with $D_{1}$ as a singular fiber and two global sections $C_{31}, C_{41}$ meeting $C$, and

$$
\varphi_{D_{2}}: X \rightarrow \mathbb{P}^{1}
$$

with $D_{2}$ as a singular fiber and two global sections $C_{21}, C_{31}$ meeting $C$.

Choose $C_{31}$ as the zero section of $\varphi_{D_{1}}$ and $C_{21}$ as the zero section of $\varphi_{D_{2}}$. We now consider the Mordell-Weil groups $\operatorname{MW}\left(\varphi_{D_{i}}\right)(i=1,2)$, that is, the group of the global sections of $\varphi_{i}$. Then MW $\left(\varphi_{i}\right)$ is an abelian subgroup of $\operatorname{Aut}(X / k)=\operatorname{Bir}(X / k)$.

Let $f_{1}$ and $f_{2}$ denote the automorphisms of $X$ given respectively by $C_{41} \in \operatorname{MW}\left(\varphi_{D_{1}}\right)$ and $C_{31} \in \mathrm{MW}\left(\varphi_{D_{2}}\right)$. As in the complex case [Ko63] (see also [DO19, Prop. 3.9]), by a result of Néron ([Ne64]), $f_{1}$ acts on

$$
C(k) \backslash(\operatorname{Sing} C)(k)=C(k) \backslash\{0, \infty\}=\mathbb{G}_{m}(k)=k^{\times}
$$

by the multiplication by $t$ and $f_{2}$ acts on

$$
C(k) \backslash(\operatorname{Sing} C)(k)=C(k) \backslash\{\infty\}=\mathbb{G}_{a}(k)=k
$$

by the addition by 1 , with respect to the affine coordinate $x$ of $C$ and the coordinate values $C \cap C_{11}=\{\infty\}, C \cap C_{21}=\{0\}, C \cap C_{31}=\{1\}, C \cap C_{41}=\{t\}$ in Definition 3.2.

In particular, both $f_{i}(i=1,2)$ preserve $C$ and the induced actions $\left.f_{i}\right|_{C} \in \operatorname{Aut}(C / k)$ on $C$ are given, under the coordinate $x$, by

$$
\left.f_{1}\right|_{C}(x)=t x,\left.f_{2}\right|_{C}(x)=x+1 .
$$

Thus

$$
\left(\left.f_{1}\right|_{C}\right)^{n} \circ\left(\left.f_{2}\right|_{C}\right) \circ\left(\left.f_{1}\right|_{C}\right)^{-n}(x)=x+t^{n}
$$

i.e., the additive translation by $t^{n}$, and therefore

$$
f_{1}^{n} \circ f_{2} \circ f_{1}^{-n} \in G(X, C, P) \text { and }\left(\left.f_{1}\right|_{C}\right)^{n} \circ\left(\left.f_{2}\right|_{C}\right) \circ\left(\left.f_{1}\right|_{C}\right)^{-n} \in \Gamma
$$

for any integer $n$. Consider the following subgroup

$$
\Gamma_{1}:=\left\langle\left(\left.f_{1}\right|_{C}\right)^{n} \circ\left(\left.f_{2}\right|_{C}\right) \circ\left(\left.f_{1}\right|_{C}\right)^{-n} \mid n \in \mathbb{Z}\right\rangle
$$

of $\Gamma$. By the description above, $\Gamma_{1}$ is isomorphic to the group $G_{t}$ in Lemma 3.1. Thus, $\Gamma_{1}$ is not finitely generated by Lemma 3.1. This completes the proof of the second assertion (2).

Let $\pi_{1}: Y_{1} \rightarrow X$ be the blow-up of $X$ at $P$ and $E_{P} \subset Y_{1}$ the exceptional curve. We choose $Q \in E_{P}(k) \backslash\left\{\left[v_{1}\right],\left[v_{2}\right]\right\}$. Here $v_{1}$ and $v_{2}$ are tangent directions of $C$ and $C_{11}$ at $P$. We then take the blow-up $\pi_{2}: Y \rightarrow Y_{1}$ of $Y_{1}$ at $Q$.

The following theorem completes the proof of Theorem 1.1 (2).

Theorem 3.9. Aut $(Y / k)$ is not finitely generated. 
Proof. As $\left.f\right|_{T_{X, P}}=\mathrm{id}_{T_{X, P}}$ for $f \in G(X, P)$, we have

$$
G(X / k, P) \subset \operatorname{Aut}(Y / k) \subset \operatorname{Aut}(X / k)
$$

via $\pi_{1} \circ \pi_{2}$. By Proposition 3.8, the group $G(X, P)$ is not finitely generated. So, if [Aut $(Y / k): G(X, P)]<\infty$, then the result follows from Theorem 2.1 .

In what follows, we prove $[$ Aut $(Y / k): G(X, P)]<\infty$. Observe that

$$
\left|K_{Y}\right|=\left\{E_{P}^{\prime}+2 E_{Q}\right\} \text {, }
$$

where $E_{P}^{\prime}$ is the proper transform of $E_{P}$ and $E_{Q}$ is the exceptional divisor of the second blow-up $Y \rightarrow Y_{1}$ at $Q$. Thus, for every $f \in \operatorname{Aut}(Y / k)$, we have

$$
f\left(E_{P}^{\prime}\right)=E_{P}^{\prime}, f\left(E_{Q}\right)=E_{Q} .
$$

Therefore, via $p_{2}$ and $p_{1}$, we can identify

$$
\operatorname{Aut}(Y / k)=\operatorname{Aut}\left(Y_{1} / k, E_{P}\right) \cap \operatorname{Aut}\left(Y_{1} / k, Q\right) \subset \operatorname{Aut}(X / k, P) .
$$

Let $f \in \operatorname{Aut}(Y / k)$. We regard $f \in \operatorname{Aut}\left(Y_{1} / k\right)$ and $f \in \operatorname{Aut}(X / k, P)$ under the identification above. Then, by Proposition [3.6, $f$ fixes $\left[v_{1}\right]$ and $\left[v_{2}\right]$ on $E_{P} \subset Y_{1}$ pointwisely. So, $\left.f\right|_{E_{P}} \in \operatorname{Aut}\left(E_{P} / k\right)$ fixes three distinct points $\left[v_{1}\right],\left[v_{2}\right], Q \in E_{p}(k)$ pointwisely. Thus $\left.f\right|_{E_{P}}=i d_{E_{P}}$, as $E_{P} \simeq \mathbb{P}^{1}$. Therefore, for $f \in \operatorname{Aut}(X / k, P)$, we have $f \in \operatorname{Aut}(Y / k)$ if and only if $\left.f\right|_{E_{P}}=i d_{E_{P}}$, that is, if and only if $d f_{P}=c(f) \operatorname{id}_{T_{X}, P}$ for some $c(f) \in k^{\times}$. Then $f^{*} \omega_{X}=c(f)^{2} \omega_{X}$ and hence

$$
G(X, P)=\operatorname{Ker}\left(\left.\alpha\right|_{\text {Aut }(Y / k)}: \operatorname{Aut}(Y / k) \rightarrow k^{\times}\right) .
$$

Here $\alpha$ is the canonical representation of $\operatorname{Aut}(X / k)$ and $\left.\alpha\right|_{\operatorname{Aut}(Y / k)}$ is the restriction of $\alpha$ to $\operatorname{Aut}(Y / k)$ under $\operatorname{Aut}(Y / k) \subset \operatorname{Aut}(X / k)$. Therefore

$$
[\operatorname{Aut}(Y / k): G(X, P)]=|\operatorname{Im} \alpha|_{\operatorname{Aut}(Y / k)}|\leq| \operatorname{Im} \alpha \mid<\infty,
$$

by Theorem 3.7. This completes the proof of Theorem 3.9.

Remark 3.10. Under terminologies of [DO19], what we proved here is nothing but the fact that $Y$ is a core surface associated to a very special triple $(X, C, P)$ over $k$.

\section{Proof of Corollary 1.2}

In this section, we shall prove Theorem 4.1. Theorem 1.1 (2) and Theorem 4.1 clearly imply Corollary 1.2 in Introduction.

Theorem 4.1. Let $k$ be the base field as in Introduction and let d be an integer such that $d \geq 3$. Choose $d-2$ integers $g_{i}(1 \leq i \leq d-2)$ such that

$$
2 \leq g_{1}<g_{2}<\ldots<g_{d-2} \text {. }
$$

Let $Y$ be a smooth projective surface in Theorem 1.1 (2) and let $C_{g_{i}}$ be a smooth projective curve of genus $g_{i}$ defined over $k$. Then

$$
Y_{d}:=Y \times C_{g_{1}} \times \ldots \times C_{g_{d-2}}
$$

is a smooth projective variety of $\operatorname{dim} Y_{d}=d$ such that Aut $\left(Y_{d} / k\right)$ is discrete and not finitely generated.

In the rest of this section, we prove Theorem 4.1.

Lemma 4.2. Both Aut $\left(C_{g_{i}} / k\right)$ and Aut $\left(Y_{d} / k\right)$ are discrete. 
Proof. By the Künneth formula, we have

$$
H^{0}\left(Y_{d}, T_{Y_{d}}\right)=H^{0}\left(Y, T_{Y}\right) \oplus H^{0}\left(C_{g_{1}}, T_{C_{g_{1}}}\right) \oplus \ldots \oplus H^{0}\left(C_{g_{d-2}}, T_{C_{g_{d-2}}}\right) .
$$

As deg $T_{C_{g_{i}}}=2-2 g_{i}<0$, it follows that $H^{0}\left(C_{g_{i}}, T_{C_{g_{i}}}\right)=0$. By our choice of $Y$, we have $H^{0}\left(Y, T_{Y}\right)=0$ as well (cf. Remark 1.3). Hence $H^{0}\left(Y_{d}, T_{Y_{d}}\right)=0$ and we are done.

Remark 4.3. There is a smooth projective surface $S$ of general type with non-zero regular global vector field over $k$ ([La83]). In particular, unlike in characteristic zero, Aut $(V / k)$, and hence Aut $((Y \times V) / k)$, could be non-discrete even if $V$ is a smooth projective variety of general type.

Set

$$
Z:=C_{g_{1}} \times \ldots \times C_{g_{d-2}}
$$

Lemma 4.4. One has

$$
\operatorname{Aut}(Z / k)=\operatorname{Aut}\left(C_{g_{1}} / k\right) \times \ldots \times \operatorname{Aut}\left(C_{g_{d-2}} / k\right)
$$

under the natural inclusion of the right hand side into the left hand side.

Proof. We prove the equality by the induction on $d-2$. If $d-2=1$, then the result is clear. Now assume $d-2 \geq 2$. Set

$$
Z^{\prime}:=C_{g_{2}} \times \ldots \times C_{g_{d-2}} .
$$

Then $Z=C_{g_{1}} \times Z^{\prime}$. We denote any closed point of $Z$ as $(x, t)$ where $x \in C_{g_{1}}$ and $t \in Z^{\prime}$.

Notice that genus does not change under any inseparable morphism. Thus, there is no non-constant morphism from $C_{g_{i}}$ to $C_{g_{j}}$ whenever $i<j$, that is, whenever $g_{i}<g_{j}$ (See eg. [Ha77, Chap IV, Sect 4.2]). Hence if $C \subset Z$ is isomorphic to $C_{g_{1}}$, then $C$ is a fiber of the projection to the second factor:

$$
\pi: Z=C_{g_{1}} \times Z^{\prime} \rightarrow Z^{\prime} ;(x, t) \mapsto t .
$$

Hence Aut $(Z / k)$ preserves $\pi$. It follows that any $F \in$ Aut $(Z / k)$, which is discrete, is of the form

$$
F(x, t)=\left(f_{t}(x), f(t)\right)
$$

where $f \in \operatorname{Aut}\left(Z^{\prime}\right)$ and $f_{t} \in \operatorname{Aut}\left(C_{g_{1}} / k\right)$ parametrized by $t \in Z^{\prime}$. As $\operatorname{Aut}\left(C_{g_{1}} / k\right)$ is discrete, it follows that $f_{t}$ does not depend on $t$. Thus

$$
\operatorname{Aut}(Z / k)=\operatorname{Aut}\left(C_{g_{1}} / k\right) \times \operatorname{Aut}\left(Z^{\prime} / k\right),
$$

and the result follows from the induction on $d-2$.

Lemma 4.5. Aut $(Z / k)$ is a finite group.

Proof. This follows from Lemma 4.4 and the fact that Aut $\left(C_{g_{i}} / k\right)$ is a finite group. The finiteness of Aut $\left(C_{g_{i}} / k\right)$ can be shown as follows. By considering pluricanonical morphisms of $C_{g_{i}}$, one can regard Aut $\left(C_{g_{i}} / k\right)$ as a Zariski closed subscheme of PGL $(N, k)$ for some positive integer $N$. As Aut $\left(C_{g_{i}} / k\right)$ is discrete (Lemma 4.2), Aut $\left(C_{g_{i}} / k\right)$ is then a reduced Zariski closed subscheme of dimension 0 of $\operatorname{PGL}(N, k)$. As $\operatorname{PGL}(N, k)$ is noetherian, it follows that $\mid$ Aut $\left(C_{g_{i}} / k\right) \mid<\infty$ as claimed.

Lemma 4.6. One has

$$
\operatorname{Aut}\left(Y_{d} / k\right)=\operatorname{Aut}(Y / k) \times \operatorname{Aut}(Z / k)
$$

under the natural inclusion of the right hand side into the left hand side. 
Proof. We have $Y_{d}=Y \times Z$. As $Y$ is birational to a K3 surface, the $m$-th canonical map $\Phi_{\left|m K_{Y_{d} \mid}\right|}$ of $Y_{d}$ with sufficiently large $m$ is nothing but the projection from $Y_{d}$ to the second factor:

$$
p: Y_{d}:=Y \times Z \rightarrow Z \text {. }
$$

From now, our proof is very close to the proof of Lemma 4.4. We denote any closed point of $Y_{d}$ as $(y, z)$ where $y \in Y$ and $z \in Z$. As Aut $(Y / k)$ preserves the $m$-th canonical map, it follows that any $G \in \operatorname{Aut}\left(Y_{d-2} / k\right)$, which is discrete, is of the form

$$
G(y, z)=\left(g_{z}(y), g(z)\right)
$$

where $g \in \operatorname{Aut}(Z)$ and $g_{z} \in \operatorname{Aut}(Y / k)$ parametrized by $z \in Z$. As Aut $(Y / k)$ is discrete, it follows that $g_{z}$ does not depend on $z$. Thus

$$
\operatorname{Aut}\left(Y_{d} / k\right)=\operatorname{Aut}(Y / k) \times \operatorname{Aut}(Z / k),
$$

as claimed.

By Lemma 4.2. Aut $\left(Y_{d} / k\right)$ is discrete. By Lemma 4.5 and Lemma 4.6, Aut $\left(Y_{d} / k\right)$ has a finite index subgroup which is isomorphic to Aut $(Y / k)$. By our choice of $Y$, the group $\operatorname{Aut}(Y / k)$ is not finitely generated (Theorem $1.1(2))$. Hence by Theorem 2.1, Aut $\left(Y_{d} / k\right)$ is not finitely generated as well. This completes the proof of Theorem 4.1.

\section{REFERENCES}

[BHPV04] Barth, W., Hulek, K., Peters, C., Van de Ven, A., : Compact complex surfaces. Second enlarged edition. Springer Verlag, Berlin-Heidelberg (2004).

[BH62] Borel, A., Harish-Chandra, : Arithmetic Subgroups of Algebraic Groups, Ann. Math. 75 (1962) $485-535$.

[BM77] Bombieri, E., Mumford, D., Enriques' classification of surfaces in char. p. II., Complex analysis and algebraic geometry, 23-42. Iwanami Shoten, Tokyo, 1977.

[Br19] Brion, M., : Notes on automorphism groups of projective varieties, 2019, available at http://www-fourier.univ-grenoble-alpes.fr/ mbrion/notes.html

[DO19] Dinh, T.-C., Oguiso, K., : A surface with discrete and nonfinitely generated automorphism group, Duke Math. J. 168 (2019) 941-966.

[Fu83] Fujita, T., : On hyperelliptic polarized varieties, Tohoku Math. J. 35 (1983) 1-44.

[Ja13] Jang, J., : The representations of the automorphism groups and the Frobenius invariants of K3 surfaces, Michigan Math. J. 65 (2016) 147-163, arXiv:1312.7634.

[Ko63] Kodaira, K., : On compact analytic surfaces. II, Ann. of Math. 77 (1963), 563-626.

[Ha77] Hartshorne, R., : Algebraic Geometry, GTM 52, Springer Verlag, New York (1977).

[Ko09] Kollár, J., : Birational rigidity of Fano varieties and field extensions, Proc. Steklov Inst. Math. 264 (2009) 96-101.

[La83] Lang, W. E., : Examples of surfaces of general type with vector fields, Arithmetic and geometry, Vol. II, 167-173, Progr. Math. 36 Birkhäuser Boston, Boston, MA, 1983.

[Le17] Lesieutre, J., : A projective variety with discrete non-finitely generated automorphism group, Invent. Math. 212 (2018) 189-211.

[LM18] Lieblich, M., Maulik, D., : A note on the Cone Conjecture for K3 surfaces in positive characteristic, Math. Res. Lett., 25 (2018) 1879-1891.

[Mu74] Mumford, D., : Abelian varieties. With appendices by C. P. Ramanujam and Yuri Manin, Corrected reprint of the second (1974) edition. Tata Institute of Fundamental Research Studies in Mathematics 5, Published for the Tata Institute of Fundamental Research, Bombay; by Hindustan Book Agency, New Delhi (2008).

[Ne64] Néron, A., : Modèles minimaux des variétés abéliennes sur les corps locaux et globaux, IHES Publ. Math. 21 (1964). 
[Og89] Oguiso, K., : On Jacobian fibrations on the Kummer surfaces of the product of non-isogenous elliptic curves, J. Math. Soc. Japan 41 (1989) 651-680.

[Og07] Oguiso, K., : Automorphisms of hyperkäahler manifolds in the view of topological entropy, Algebraic geometry, Contemp. Math. 422 (2007) 173-185.

[PS12] Prendergast-Smith, A. : The cone conjecture for abelian varieties, J. Math. Sci. Univ. Tokyo 19 (2012) 243-261.

[RS76] Rudakov, A. N., Shafarevich, I.R., : Inseparable morphisms of algebraic surfaces, Izv. Akad. Nauk SSSR Ser. Mat. 40 (1976) 1269-1307.

[Sh75] Shioda, T., : Algebraic cycles on certain K3 surfaces in characteristic p, Manifolds-Tokyo 1973, 357-364, Univ. Tokyo Press (1975).

[St85] Sterk, H., : Finiteness results for algebraic K3 surfaces, Math. Z. 189 (1985) 507-513.

[Su82] Suzuki, M., : Group theory. I, Grundlehren der Mathematischen Wissenschaften, 247, SpringerVerlag, Berlin-New York, 1982.

[Ue75] Ueno, K., : Classification theory of algebraic varieties and compact complex spaces. Lecture Notes in Mathematics, 439, Springer-Verlag, 1975.

[Wa19] Wang, L., : On automorphisms and the cone conjecture for Enriques surfaces in odd characteristic, arXiv:1908.07928

Mathematical Sciences, the University of Tokyo, Meguro Komaba 3-8-1, Tokyo, Japan, and National Center for Theoretical Sciences, Mathematics Division, National Taiwan University, TAIPEI, TAIWAN

E-mail address: oguiso@ms.u-tokyo.ac.jp 